\title{
Evolución del grado de afectación del nervio facial con fisioterapia en un instituto especializado en Lima
}

\author{
Evolution of the degree of facial nerve involvement with physiotherapy in a specialized institute in Lima \\ Patricia Andrea Fuentes Lozada ${ }^{1}$, Diana Lizette Valenzuela Rodríguez ${ }^{1}$, Julissa Estefany Yesang Canales ${ }^{1}$, \\ Adela Martínez Ampuero ${ }^{1,2}$, Jorge Gabriel Marroquín Ballón ${ }^{3}$, Paúl Rubén Alfaro Fernández ${ }^{4}$
}

\section{RESUMEN}

La fisioterapia es fundamental en el tratamiento de la parálisis facial, la escala House-Brackmann 2.0 registra y evalúa la afectación del nervio facial, haciendo un análisis en la funcionalidad, simetría facial y sincinesias, permitiendo observar los cambios en su evolución. Objetivo: Registrar los cambios presentes en pacientes con parálisis facial periférica luego de un tratamiento estándar por un determinado tiempo en un Instituto Especializado. Material y Métodos: Estudio observacional, descriptivo tipo cohorte única, longitudinal y prospectivo que evaluó a todos los pacientes adultos con parálisis facial periférica que asistieron al servicio de Neurorrehabilitación del INCN, cumpliendo con los criterios de inclusión. El instrumento utilizado fue la escala House-Brackmann 2.0, la cual se aplicó en cuatro oportunidades (primer día de evaluación, primer mes, segundo mes y tercer mes). Resultados: Se observó que el puntaje promedio del grado de afectación del nervio facial antes del tratamiento fue 18,82 (IC: 11-24)/ Grado IV; en la segunda evaluación, aún con los 72 pacientes, disminuyó a 10,87 (IC: 5-21)/Grado III; a la tercera llegaron 65 pacientes con 6,32 (IC: 4-14)/Grado II y finalmente a la cuarta llegaron 23 pacientes con 4,26 (IC: 4-7)/ Grado I. Conclusiones: El 97,2\% de pacientes que iniciaron su tratamiento presentando un grado IV culminaron en grado I logrando la simetría facial y el fortalecimiento muscular, según la escala House-Brackmann 2.0.

PALABRAS CLAVE: House-Brackmann Facial Nerve Grading System 2.0, Peripheral facial paralysis, Phisiotherapy.

\section{SUMMARY}

The physiotherapy is essential in the treatment of facial paralysis, the House-Brackmann 2.0 scale records and evaluates the involvement of the facial nerve, making an analysis of functionality, facial symmetry and synkinesis, allowing to observe the changes in their evolution. Objective: To record the changes present in patients with peripheral facial paralysis after a standard treatment for a certain time in a Specialized Institute. Material and Methods: Observational, descriptive single cohort type, longitudinal and prospective study that evaluated all adult patients with peripheral facial paralysis who attended the Neurorehabilitation service of the INCN, fulfilling the inclusion criteria. The instrument used was the House-Brackmann 2.0 scale, which was applied four times (first day of evaluation, first month, second month and third month). Results: It was observed that the average score of

\footnotetext{
Escuela de Tecnología Médica, Facultad de Medicina, Universidad Peruana Cayetano Heredia. Lima, Perú Instituto Nacional De Rehabilitación "Dra. Adriana Rebaza Flores” Amistad Perú - Japón. Lima, Perú. Instituto Nacional de Ciencias Neurológicas. Lima, Perú

Universidad de San Martín de Porres. Lima, Perú
} 
the degree of the facial nerve involment before treatment was 18,82 (CI:11-24/Grade IV); In the second evaluation, even with the 72 patients, it decreased to 10,87 (CI: 5-21/Grade III); 65 patients arrived at the third with 6,32 (CI: 4-14/Grade II) and finally 23 patients with 4,26 (CI: 4-7/Grade I) arrived at the fourth. Conclusions: 97,2\% of patients who started their treatment presenting a grade IV, culminated in grade I achieving facial symmetry and muscle strengthening, according to the House-Brackmann 2.0 scale.

KEYWORDS: House-Brackmann Facial Nerve Grading system 2.0, Peripheral facial paralysis, Phisiotherapy.

\section{INTRODUCCIÓN}

El nervio facial (VII par craneal) presenta un aproximado de 7000 fibras, en su mayoría, motoras (1); una lesión en cualquier parte de su recorrido genera una parálisis facial. Se clasifica en parálisis central y periférica. El daño del núcleo del nervio facial produce parálisis facial periférica, afectando a los músculos faciales del mismo lado de la lesión y presentando alteraciones lagrimales y salivales (2). Seddón estableció la clasificación histopatológica de toda lesión nerviosa periférica: neuropraxia (leve), axonotmesis (moderada) y neurotmesis (severa) (3).

La parálisis facial de Bell es la más común, aparece de forma aguda y brusca, los surcos de expresión desaparecen en el lado afectado y se acentúan en el lado contrario. Dentro de su pronóstico hay una recuperación en el $85 \%$ de los afectados y un $15 \%$ de casos presentan secuelas (4). Su diagnóstico se basará en la clínica, asimetría e inmovilidad facial y pruebas como la electromiografía que constatan el grado de daño en el nervio. El tratamiento es farmacológico y fisioterapéutico (5).

La termoterapia es un agente físico que utiliza el calor generando respuestas fisiológicas en beneficio del paciente (6). La masoterapia y los ejercicios fisioterapéuticos son técnicas manuales que van dirigidos a los receptores propioceptivos (huso neuromuscular y aparato tendinoso de Golgi) con el objetivo de mantener y mejorar el tono y trofismo muscular (7).

En la actualidad, la fisioterapia basa su tratamiento en el movimiento facial específico y selectivo, buscando el reclutamiento no de masas musculares, sino de unidades motoras funcionales. A nivel de reeducación neuromuscular se basa en la teoría de la neuroplasticidad cerebral y en la capacidad humana de adaptación de nuevas conductas motoras (8).

Una de las escalas para evaluar el grado de afectación del nervio facial es la escala House Brackmann, validada en 1985 (9), y actualizada en el año 2009, llamándose House-Brackmann Facial Nerve Grading System 2.0 (10); esta incorpora nuevas valoraciones sobre el movimiento facial dividiéndolo en 4 regiones: frente, ojo, surco naso labial y comisura bucal; especificando una gradación de 1-6 puntos agrupándolo en 6 categorías en base a una valoración cuantitativa y cualitativa, así como los movimientos secundarios en una gradación de 0-3 puntos. La Universidad Nacional de Chimborazo (2017 Ecuador) (1), Universidad Nacional Mayor de San Marcos (2015 - Perú) (11), La Universidad Estácio de Sergipe (2018 - Brasil) (12), entre otras, utilizaron en su estudio la escala mencionada como herramienta de medición del grado de afectación del nervio facial.

En el año 2014, en Lima (Perú), se publicó la Resolución Directoral emitida por el Instituto Nacional de Ciencias Neurológicas (INCN) y refrendada por el Ministerio de Salud; en ella se resuelve aprobar la "Guía práctica clínica de parálisis facial periférica”, donde se recomienda la aplicación de termoterapia, masoterapia y ejercicios terapéuticos, el uso de férula palpebral en caso de lagoftalmos y queda contraindicado el uso de vibradores y goma de mascar (13). La parálisis facial periférica es considerada un problema de salud mundial generando una discapacidad social en la participación con su entorno (14), hasta la fecha mantiene una alta incidencia, de 30 a 40 casos por cada 100000 personas (15).

En el INCN (Perú), registraron más de 1500 casos entre los años 2002 y 2006, en su mayoría axonotmesis, en el 2014 hubo un registro de 643 casos con una prevalencia de los grados IV y $\mathrm{V}$ según la escala House-Brackmann 2.0 (11) y en el año 2016 se registraron 1168 casos (16).

El objetivo principal del estudio fue registrar los cambios presentes en pacientes con parálisis facial periférica luego de un tratamiento convencional por un determinado tiempo en un Instituto Especializado. Así mismo, los objetivos específicos fueron verificar la aplicación del tratamiento de termoterapia, masoterapia y ejercicios enfocado en la parálisis facial periférica para recuperar el fortalecimiento muscular 
de la hemicara afectada y lograr una simetría facial, y describir los cambios encontrados de la 1era, 2da, 3era y 4ta evaluación según la escala de House-Brackmann 2.0 en pacientes con parálisis facial periférica.

\section{MATERIAL Y MÉTODOS}

El estudio fue de tipo observacional, descriptivo, de cohorte única prospectiva, ya que no presentó un grupo control y solo se midió la evolución del efecto(17). La población de estudio fueron todos los pacientes adultos que ingresaron al departamento de Neurorrehabilitación presentando parálisis facial periférica de Bell. El tamaño muestral fue calculado por medio de la calculadora GRANMO con un riesgo alfa de 0.05 , un tipo de contraste bilateral y un riesgo beta de 0.20 ; la proporción con acontecimiento preintervención es $82 \%$, la proporción con acontecimiento post-intervención es $95 \%$ y la proporción prevista de pérdida de seguimiento es $10 \%$, obteniendo como resultado 95 pacientes (18). El muestreo fue no probabilístico por conveniencia, ya que los pacientes se fueron reclutando de acuerdo con la llegada al servicio de Fisioterapia hasta alcanzar la muestra proyectada. Sin embargo, durante la recolección de datos, la realidad en el servicio de Neurorrehabilitación varió en cuanto al cálculo esperado, disminuyendo el número a un total de 72 pacientes.

Los criterios de inclusión fueron: todos los pacientes con parálisis facial periférica de Bell (tipo: axonotmesis) en etapa aguda, diagnosticados por la prueba de electromiografía, que reciban intervención únicamente en el Instituto Nacional de Ciencias Neurológicas, y que aceptaran firmar el consentimiento informado para participar en el estudio.

En cuanto a los criterios de exclusión, fueron: pacientes con parálisis facial central, con niveles de lesión de tipo neuropraxia y neurotmesis, que presenten segundo evento de PFP, alteraciones cognitivas y/o mentales, que lleven terapia particular adicional o con parálisis facial bilateral.

Con respecto a procedimientos y técnicas, antes del inicio del tratamiento se informó al paciente sobre el estudio, si aceptaba su participación firmaba el consentimiento informado, él y el investigador. Durante la investigación, el encargado de aplicar el tratamiento convencional fue el fisioterapeuta, como investigadoras nos encargamos de medir y registrar los cambios a nivel motor y funcional del paciente utilizando la escala de House-Brackmann 2.0. En la primera sesión se utilizó una ficha de recolección de datos, permitiéndonos tener un conocimiento del estado actual del participante antes de dar inicio a la evaluación y el tratamiento correspondiente. Toda ficha fue guardada mediante un código personalizado manteniendo su confidencialidad. Luego se contó con la escala de gradación House-Brackmann 2.0 para evaluar el grado de afectación del nervio facial. En el presente estudio, la medición se realizó en cuatro oportunidades (el primer día de evaluación, primer mes, segundo mes y tercer mes). Se dio inicio al tratamiento convencional con la aplicación de compresas húmedas calientes $(\mathrm{CHC})$ en ambas hemicaras, luego el paciente se ubicó en una silla frente al espejo para que el fisioterapeuta, situado detrás de él, inicie con masoterapia (Eufflerage - masaje estimulante, Petrissage - masaje relajante); luego con ejercicios terapéuticos de facilitación neuromuscular, como también movimientos de enfatización gesticular, la intención de que el paciente esté frente a un espejo fue con el propósito de estimular la retroalimentación visual mejorando la contracción concéntrica de los músculos afectados. Cada procedimiento duró 15 minutos aproximadamente.

Para el plan de Análisis, la base de datos fue almacenada en Microsoft Excel, en donde se clasificó con codificación para el procesamiento de datos asignándole un valor numérico a las variables presentadas. Así mismo, se realizaron tablas de frecuencia y porcentaje en las variables cualitativas y se calculó la media, mediana y desviación estándar en las variables cuantitativas.

Esta investigación se sustentó en los principios éticos que justifica la misma: beneficencia, no maleficencia, justicia y autonomía. El paciente no presentó riesgo de daño a su integridad física ni mental al momento de participar en esta investigación, debido a que los registros de datos se llevaron a cabo bajo un procedimiento rutinario (evaluación y tratamiento convencional).

\section{RESULTADOS}

Participaron un total de 81 pacientes con parálisis facial periférica, 9 fueron retirados debido a no completar con las 4 evaluaciones señaladas, quedando 72 participantes. El sexo masculino tuvo la mayor proporción $(59,7 \%)$. El mayor porcentaje corresponde al grupo de casados (52,8\%); un 50\% tuvo ocupación dependiente. El lado afectado con mayor proporción fue el izquierdo (56,9\%). La edad promedio fue de 
Tabla 1. Variables sexo, estado civil, ocupación, lado afectado y edad en los participantes con parálisis facial.

\begin{tabular}{lccc}
\hline VARIABLES & & $\mathbf{n}$ & $\mathbf{\%}$ \\
\hline \multirow{2}{*}{ Sexo } & Femenino & 29 & 40,3 \\
& Masculino & 43 & 59,7 \\
& Soltero & 28 & 38,9 \\
Estado civil & Casado & 38 & 5,8 \\
& Viudo & 3 & 4,2 \\
& Divorciado & 3 & 4,2 \\
& & & \\
& Dependiente & 36 & 50 \\
Ocupación & Independiente & 19 & 26,4 \\
& Ama de casa & 11 & 15,3 \\
& Estudiante & 5 & 6,9 \\
& Sin ocupación & 1 & 1,4 \\
Lado afectado & & & 43,1 \\
& Derecho & 31 & 56,9 \\
Edad & Izquierdo & 41 & Rango \\
& & & $19-79$ \\
\hline
\end{tabular}

Tabla 2. Presencia de dolor y EVA en los participantes con parálisis facial.

\begin{tabular}{lccc}
\hline VARIABLES & Escala & $\mathbf{N}$ & $\mathbf{\%}$ \\
\hline \multirow{2}{*}{ Presencia de dolor } & $\mathrm{Si}$ & 39 & 54,2 \\
& No & 33 & 45,8 \\
& & & \\
& 0 & 33 & 45,8 \\
& 1 & 1 & 1,4 \\
& 2 & 3 & 4,2 \\
EVA dolor & 3 & 2 & 2,8 \\
& 4 & 3 & 4,2 \\
& 5 & 8 & 11,1 \\
& 6 & 6 & 8,3 \\
& 7 & 6 & 8,3 \\
& 8 & 6 & 8,3 \\
& 9 & 1 & 1,4 \\
& 10 & 3 & 4,2 \\
\hline
\end{tabular}

45,82 años (DE: 1,7), donde el menor y mayor rango de edad corresponde a 19 y 79 años respectivamente (tabla 1). El 54,2\% presentó dolor, con un $11,1 \%$ en dolor moderado según EVA DOLOR. (tabla 2). Con respecto a los resultados de la EMG, el $57 \%$ obtuvo axonotmesis moderada; 40,2\%, axonotmesis leve y 2,8\%, axonotmesis severa (tabla 3). El promedio del número de sesiones recibidas fue 23,39 (DE: 6.3), con un mínimo de 15 sesiones y un máximo de 36 sesiones (tabla 4). 
Tabla 3.- Tipo de Axonotmesis según EMG en los participantes con parálisis facial.

\begin{tabular}{cccc}
\hline VARIABLE & & $\mathbf{N}$ & $\mathbf{\%}$ \\
\hline \multirow{3}{*}{ Axonotmesis } & Leve & 29 & 40,2 \\
& Moderada & 41 & 57 \\
& Severa & 2 & 2,8 \\
\hline
\end{tabular}

Tabla 4. Número de sesiones en los participantes con parálisis facial.

\begin{tabular}{lc}
\hline & $\mathbf{n}$ \\
\hline Media & 23,39 \\
Mediana & 24 \\
Rango mínimo & 15 \\
Rango máximo & 36 \\
Desviación estándar & 6,3 \\
\hline
\end{tabular}

En cuanto a la Evolución, de la primera a la segunda evaluación se mantuvo el número de participantes (72 pacientes), sin embargo, se empezaron a notar cambios de mejoría. El puntaje promedio de la primera evaluación fue 18,82 (IC: 11-24) / Grado IV, mientras que en la segunda disminuyó a un 10,87 (IC: 5-21) / Grado III, es decir, cambió de "disfunción moderadamente grave" a "disfunción moderada". El 9,7\% (7 pacientes) se recuperó antes de completarse el segundo mes. A la tercera evaluación llegaron 65 pacientes con un puntaje promedio de 6,32 (IC: 4-14) / Grado II, es decir, varió benéficamente a una "disfunción leve", recuperándose el 30,5\% (22 pacientes) quedando 43 pacientes. El 27,8\% (20 pacientes) se recuperó antes de llegar a la cuarta evaluación, quedando 23 pacientes, con un puntaje promedio de 4,26 (IC: 4-7) / Grado I, es decir, varió benéficamente a "normal", en donde se recuperó el $29,2 \%$ (21 pacientes) quedando 2 pacientes (tabla 5, tabla y tabla 7).

Al término del tiempo establecido, se observó que, de la primera a la cuarta evaluación, de los 72 participantes hubo una recuperación del 97,2\%. Solo dos pacientes quedaron con una puntuación 7 (Grado II) a la espera de una reevaluación (gráfico 1).

\section{DISCUSIÓN}

La fisioterapia es fundamental en el tratamiento de esta patología, mejorando su funcionalidad y calidad de vida, utilizando en sus inicios los ejercicios faciales como primera intervención (19). Dentro de los tratamientos para la rehabilitación del paciente destacan los métodos y técnicas fisioterapéuticas, como son la facilitación neuromuscular propioceptiva, ejercicios de énfasis en mímica facial y la retroalimentación visual y auditiva, apoyado de agentes físicos y electrofísicos

Tabla 5. Evolución del grado de afectación del nervio facial entre la 1era y 2da evaluación según la escala House-Brackmann 2.0

\begin{tabular}{cccccc}
\hline & TOTAL & Media & $\begin{array}{c}\text { Desv. } \\
\text { Desviación }\end{array}$ & $\begin{array}{c}\text { Puntaje } \\
\text { mínimo }\end{array}$ & $\begin{array}{c}\text { Puntaje } \\
\text { máximo }\end{array}$ \\
\hline Puntaje - 1era EVA & 72 & 18,82 & 3,632 & 11 & 24 \\
Puntaje - 2da EVA & 72 & 10,87 & 3,918 & 5 & 21 \\
\hline
\end{tabular}

Tabla 6. Evolución del grado de afectación del nervio facial entre la 1era, 2 da y 3era evaluación según la escala House-Brackmann 2.0

\begin{tabular}{cccccc}
\hline & TOTAL & Media & $\begin{array}{c}\text { Desv. } \\
\text { Desviación }\end{array}$ & $\begin{array}{c}\text { Puntaje } \\
\text { mínimo }\end{array}$ & $\begin{array}{c}\text { Puntaje } \\
\text { máximo }\end{array}$ \\
\hline Puntaje - 1era EVA & 65 & 19,22 & 3,435 & 11 & 24 \\
Puntaje - 2da EVA & 65 & 11,46 & 3,649 & 5 & 21 \\
Puntaje - 3era EVA & 65 & 6,32 & 2,507 & 4 & 14 \\
\hline
\end{tabular}


Tabla 7. Evolución del grado de afectación del nervio facial entre la 1era, 2da, 3era y 4ta evaluación según la escala House-Brackmann 2.0

\begin{tabular}{cccccc}
\hline & TOTAL & Media & $\begin{array}{c}\text { Desv. } \\
\text { Desviación }\end{array}$ & $\begin{array}{c}\text { Puntaje } \\
\text { mínimo }\end{array}$ & $\begin{array}{c}\text { Puntaje } \\
\text { máximo }\end{array}$ \\
\hline Puntaje - 1era EVA & 23 & 20,96 & 2,383 & 17 & 24 \\
Puntaje - 2da EVA & 23 & 13,7 & 3,747 & 8 & 21 \\
Puntaje - 3era EVA & 23 & 8,48 & 2,695 & 5 & 14 \\
Puntaje - 4ta EVA & 23 & 4,26 & 0,945 & 4 & 7 \\
\hline
\end{tabular}

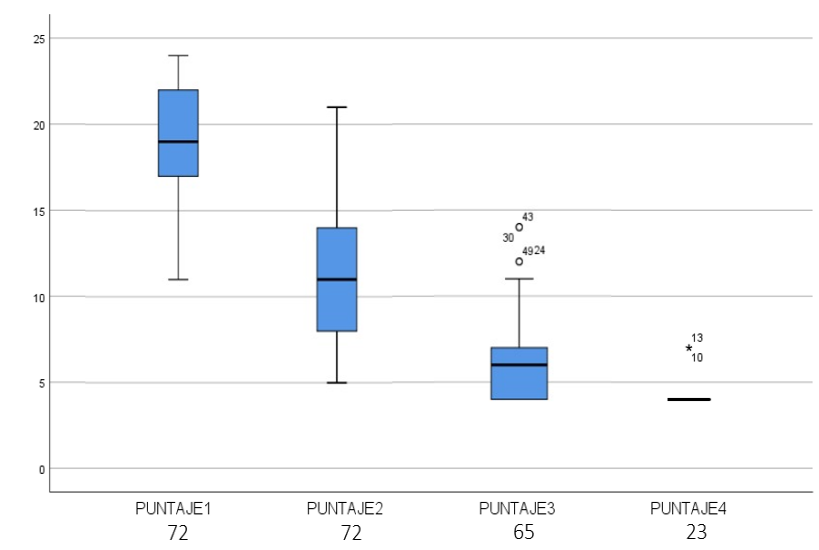

Gráfico 1. Distribución del puntaje de la escala House - Brackmann 2.0 en la 1era, 2da, 3era y 4ta evaluación, en los participantes con parálisis facial.

(12). Hernán Kabat refiere que los ejercicios de facilitación obtienen respuestas específicas del sistema neuromuscular mediante la estimulación de los propioceptores orgánicos (20). Así mismo, Paredes L. menciona que la termoterapia favorece la nutrición tisular y metabolismo celular. (21). Además, el Instituto Nacional de Trastornos Neurológicos y Accidentes Cerebrovascular, respalda los métodos de termoterapia, masoterapia y ejercicios terapéuticos (22), utilizados en el tratamiento convencional.

Por medio del presente estudio, se registraron los cambios de la afectación nerviosa en los pacientes con parálisis facial periférica a lo largo del tratamiento convencional (termoterapia, masoterapia y ejercicios terapéuticos), el promedio según el puntaje del grado de afectación del nervio facial, antes y después del tratamiento convencional, fue 18,8 (Grado IV) y 4,26 (Grado I) respectivamente, recuperándose en su totalidad el $97,2 \%$ de participantes, quedando el 2,8\% en grado II. Estos porcentajes se contrastan con el estudio descrito por Molina G. y Ortiz A., en donde se utilizaron los ejercicios fisioterapéuticos faciales en 40 pacientes obteniendo una recuperación del $65,5 \%$ del total de participantes, quedando un $32,5 \%$ en grado II (1); así mismo, Delgado M. evaluó a 96 pacientes durante 3 meses de tratamiento fisioterapéutico donde además se aplicó magneto y laserterapia, finalizando el estudio con un $68 \%$ en Grado I, un $29 \%$ en Grado II y un $3 \%$ en Grado III (23).

El 40,2\% tuvo una recuperación al finalizar el segundo mes de tratamiento y el 57\% se recuperó al finalizar el tercer mes de tratamiento, a excepción de un hombre y una mujer que iniciaron con un puntaje 24 (Grado VI) y presentaron una axonotmesis severa. Ambos obtuvieron en su cuarta evaluación una puntuación 7 (Grado II), además uno de ellos presentó estrés y el otro obesidad, y según Rodríguez (24) y Aboytes (25), estas dos condiciones se consideran perjudiciales en la recuperación de la parálisis facial, por ello el tiempo de recuperación fue más extenso en comparación a los demás participantes que culminaron su tratamiento en dos y tres meses, sin 
embargo, fueron dados de alta al recibir un bloque de sesiones adicionales. Hubo una variación de 15 a 36 sesiones, lo cual puede ser explicado debido al grado de axonotmesis (26), ya que aquellos que presentaron una axonotmesis leve $(40,2 \%)$, se recuperaron en un tiempo menor a los que presentaron una axonotmesis moderada (57\%), quedando $2(2,8 \%)$ pacientes que presentaron axonotmesis severa. El tiempo de respuesta favorable al tratamiento fue mayor al del estudio de Conceição A., en donde se observaron mejorías a partir de los 21 días con una recuperación a los dos meses (12), así mismo, Martin B. (27) refiere en su estudio una mejoría a los 15 días de haber iniciado tratamiento y una recuperación completa del $95 \%$ de pacientes a los 3 meses.

La Touche et al. recomendaron profundizar más sobre el número de sesiones por semana y tiempo de intervención (19), Conceição presentó un tratamiento de 50 minutos realizado dos veces por semana durante dos meses (12), así mismo el estudio realizado en el INCN incluye un abordaje de 45 minutos realizado 3 veces por semana durante dos y tres meses, cabe resaltar que el tiempo de mejoría depende también de la edad y tipo de lesión del nervio.

Datos encontrados refieren una prevalencia del sexo femenino (28), así mismo, los autores indican mayor frecuencia entre los 20 a 40 años y adultos mayores (29). En el caso del presente estudio, el rango de edad guarda similitud con estas investigaciones, por el contrario, al sexo, donde hubo mayor proporción del sexo masculino. El lado izquierdo tuvo mayor prevalencia y el dolor a nivel del agujero estilomastoideo se presentó durante los primeros 7 días de haberse iniciado la lesión, estos datos se corroboran con los hallazgos descritos en el estudio de Aboytes (25); es preciso señalar que los pacientes iniciaron su tratamiento con una semana de evolución y sin presentar dolor.

Según los autores Lorenzo-Cáceres I. (30) y Santa et al., (31), la electroestimulación interfiere en la regeneración nerviosa acelerando los procesos de reinervación aberrante, considerándose contraindicado para el tratamiento, sin embargo, existen centros asistenciales (32),que en la actualidad la usan como parte de su terapia convencional (32), así mismo, Delgado manifiesta que los tratamientos realizados en centros fisioterapéuticos son más efectivos que los ejercicios y masajes realizados en casa por el mismo paciente (23).
La recuperación de la parálisis facial depende de diversos tratamientos aplicados, incluyendo los métodos y técnicas empleadas por el fisioterapeuta (12), por otro lado, el grado de lesión nerviosa e inicio tardío del tratamiento se consideran determinantes en el pronóstico de recuperación del paciente (28). Según revisiones, una neuropraxia tiene un tiempo de recuperación promedio de 15 días, incluso se recuperan sin tratamiento, a comparación de una axonotmesis que dura un promedio de 1 mes a 1 año, dependiendo del grado o tipo lesión (29), sin embargo, independientemente del grado de lesión la fisioterapia es importante, pues al no haber ningún tipo de abordaje los síntomas se presentarán durante mayor tiempo (28); Monini et al., concluyen en su estudio, que los pacientes con tratamiento fisioterapéutico tuvieron 20 veces más probabilidades de mejorar su funcionalidad en un tiempo menor a los que no recibieron la intervención (33).

Desde el primer momento que llega el paciente con parálisis facial, se debe realizar una evaluación fisioterapéutica y esta debe ser periódicamente hasta su última sesión, asímismo se le brindará recomendaciones con el fin de prevenir complicaciones como espasmos musculares, sincinesias, úlceras corneales, entre otras que se pudieran presentar durante la evolución de su recuperación.

En conclusión, el 97,2\% de pacientes que iniciaron su tratamiento presentando un grado IV culminaron en grado I logrando la simetría facial y el fortalecimiento muscular, según la escala House-Brackmann 2.0. De la primera a la segunda evaluación se encontraron cambios de mejoría, con una evolución del grado IV al III. De la segunda a la tercera evaluación se registró una evolución del grado III al II. De la tercera a la cuarta evaluación se registró una evolución del grado II al I, según la escala House-Brackmann 2.0. El 2,8\% que tuvieron estrés y obesidad finalizaron su cuarta evaluación con un grado II.

Declaración de interés: Los autores declaran no tener ningún conflicto de interés en el presente trabajo de investigación.

\section{Correspondencia}

Patricia Andrea Fuentes Lozada

patricia.fuentes.1@upch.pe

Diana Lizette Valenzuela Rodríguez

diana.valenzuela.r@upch.pe 
Julissa Estefany Yesang Canales

julissa.yesang.c@upch.pe

\section{REFERENCIAS BIBLIOGRÁFICAS}

1. Molina G, Ortiz A. Ejercicios fisioterapéuticos faciales para la recuperación de pacientes en parálisis facial periférica que acuden al área de terapia física del centro de salud tipo C "Espoch-Lizarzaburu" Julio - diciembre 2016. Tesis de Licenciatura. Riobamba. Ecuador: Universidad Nacional De Chimborazo; 2017.

2. Luque C, Guevara S, Buforn A. Parálisis facial. Tesis Doctoral. Málaga, España: Hospital Clínico Universitario "Virgen Victoria"; 2016.

3. Epprecht P, Novas E, Mas S. Libro virtual de formación de otorrinolaringología [Internet]. Madrid: SEORL; 2015. (Citado el 20 de noviembre de 2017). Disponible en: https://booksmedicos.org/librovirtual-de-formacion-en-otorrinolaringologia-seorl/.

4. Saborío IE, Villalobos D, Bolaños C. Abordaje de la parálisis de Bell diagnóstico y tratamiento .Rev Med Sinergia. 2019; 4 (6): 81-89.

5. Álvarez CA, Mora N, Gonzales R. Parálisis periférica: enfoque desde la medicina física y rehabilitación en Costa Rica. Rev Med CR CA. 2015; 72 (615): 249 255.

6. Sánchez VC. Eficacia de Taping Neuromuscular en pacientes con Parálisis de Bell atendidos en la Maternidad de María, entre Agosto- Octubre 2017. Tesis Licenciatura. Chimbote,Perú: Universidad San Pedro; 2017.

7. Prats VE. Efectividad del tratamiento rehabilitador e pacientes con parálisis del nervio facial post parotidectomia y su impacto en la calidad de vida. Tesis Doctoral. Sevilla, España: Universidad de Sevilla; 2015.

8. Quesada P, López D, Quesada JL. Tratamiento rehabilitador de la Parálisis Facial Periférica. En: Junyent J, Pedro A, García A, editores. Facial Periférica. Complicaciones y secuelas. Vol. 1.3 ed. Badalona: Ediciones Medicas S.L; 2010. p. 211-216.

9. Quesada P, López D, Quesada JL. Evaluación de la parálisis facial periférica. En: Galletti F. Galleti B. Freni F, editores 1 Periférica. Complicaciones y secuelas. Vol. 1.3 ed. Badalona: Ediciones Medicas S.L; 2010. p. 183-190.

10. Bohoyo C. Ejercicios activos en la parálisis facial. Tesis Licenciatura. Pamplona, España: Universidad Pública de Navarra; 2013.

11. Cruz RL. Características de los grados de afectación del nervio facial y la discapacidad facial en pacientes con parálisis facial periférica de un año de evolución. Instituto Nacional de Ciencias Neurológicas MarzoJunio 2015.Tesis Licenciatura. Lima, Perú:
Universidad Nacional Mayor De San Marcos; 2015.

12. Conceição AD, Pereira W, Andrade E. Intervenção fisioterapêutica no tratamento de paciente com paralisia facial periférica: estudo de caso. Rev Saúde e Pesquisa. 2018; 11(1): 179-189.

13. Ministerio de Salud. Guía práctica clínica de parálisis facial periférica, del departamento de emergencia. Lima: INCN; 2014. (Citado el 20 de noviembre de 2017). Disponible en: https://www.incn.gob.pe/ servicios/neurologia/neurorrehabilitacion.

14. Benítez S, Danilla S, Troncoso E, Moya A, et al. Manejo integral de la parálisis facial. Rev Méd Clínica Las Condes. 2016; 27(1): 22-28.

15. Cáceres E, Morales M, Wulfsohn G, Montes S. Parálisis Facial Periférica. Incidencia y etiología. Rev Faso.2018; 25(1): 8-13.

16. Ministerio de Salud; Oficina De Estadística E Informática/Unidad De Estadística. Estadísticas de morbimortalidad. Lima: Ministerio de Salud; 2016.p. 21-65.

17. Hernández R, Fernández C, Baptista P. Metodología de la Investigación. 6 ed. Ciudadad de Mexico: McGraw-Hill Interamericana; 2018.

18. Calculadora de Grandària Mostral GRANMO. Proporcions: Dos proporcions independents.Madrid: IMIM; 2012 (Citado el 20 de noviembre de 2017). Disponible en: https://www.imim.cat/ofertadeserveis/ software-public/granmo/.

19. La Touche R, Escalante K, Linares M, Mesa J. Efectividad del tratamiento de fisioterapia en la parálisis facial periférica Revisión sistemática. Rev Neurol. 2008; 46(12): 714-718.

20. Lopez L. Eficacia del Método Kabat y de Terapia del espejo para mejorar la calidad de vida en pacientes amputados por diabetes mellitus, Hospital Almanzor Aguinaga Asenjo, Chiclayo-2017. Tesis Doctoral. Lambayeque,Perú: Universidad Nacional Pedro Ruiz Gallo; 2017.

21. Paredes LI. Tratamiento fisioterapéutico en parálisis facial periférica. Tesis Licenciatura. Lima, Perú: Universidad Inca Garcilaso de la Vega; 2018.

22. National Institute of Neurological Disorders. Bell's Palsy Fact Sheet. Washington DC, Estados Unidos: National Institute of and Stroke; 2018. (Citado el 20 de noviembre de 2017). Disponible en: https:// www.ninds.nih.gov/Disorders/Patient-CaregiverEducation/Fact-Sheets/Bells-Palsy-FactSheet\#3050 6

23. Delgado M, Sánchez M, Díaz A, Parson G, González Y. Tratamiento combinado de campo magnético, láser, masaje y ejercicio en la parálisis facial periférica idiopática. Acta. Fisioterapia. 2012; 34(3): 99-104.

24. Rodríguez KV, Torres E, Rodríguez M. Efecto del estrés en las sincinesias en pacientes con parálisis facial periférica idiopática crónica. Rev Lat de Med Cond. 2016; 7(1): 9-15. 
25. Aboytes C, Morales M, Torres J, Valenzuela A. Parálisis facial periférica idiopática y su asociación con el síndrome metabólico y sus componentes. Rev Mexica de Neurociencia. 2016; 17(4): 8-16.

26. Universidad Complutense de Madrid. Lesiones de los nervios periféricos. Madrid: UCM; 2014. (Citado el 20 de noviembre de 2017). Disponible en: https:// www.ucm.es/data/cont/docs/420-2014-03-28-13\%20 Lesiones\%20de\%201os\%20nervios\%20perifericos. pdf

27. Piñero B, Pérez E, Yumar A, Hernández M, Lamarque V, Castillo E. Efectividad de la rehabilitación en la parálisis de Bell. Rev Cub de Med Fís y Rehab. 2017; 9(1):1-14.

28. Rosales M. EL kinesio taping como complemento del tratamiento convencional de la parálisis facial periférica idiopática en pacientes del Patronato Municipal de Amparo Social de Latacunga. Tesis Licenciatura. Ambato,Ecuador: Universidad Técnica de Ambato; 2015.

29. Ronthal M. Greenstein P. Bell's palsy: Treatment and prognosis in adults. JM ed. UpToDate Inc. 2018.

30. Lorenzo - Cáceres I. Proyecto de tratamiento fisioterapéutico en pacientes con parálisis facial periférica. Tesis Licenciatura. Alcalá de Henares, España: Universidad de Alcalá; 2013.

31. Santa CA, Bergo B, Ferreira J. Paralisia facial de Bell e estratégias de intervenção fisioterápica preventiva ao acometimento da sincinesia. Rev Digital Buenos Aires. 2014; 19(199).

32. Vargas MH. Eficacia de un protocolo de tratamiento fisioterapéutico en pacientes con parálisis facial periférica en etapa subaguda, que acuden al Departamento de Fisiatría Del Hospital Provincial General Docente Riobamba en el periodo de inicio abril a septiembre del 2013. Tesis Licenciatura. Riobamba, Ecuador: Universidad Nacional De Chimborazo; 2013.

33. Monini S, Iacolucci C, Di Traglia M, Lazzarino A, Barbara M. Role of Kabat rehabilitation in facial nerve palsy: a randomised study on severe cases of Bell's palsy. Acta Otorhinolaryngol Ital. 2016; 36 (4):282-288.

Recibido: 24/02/2021 Aceptado: 14/05/2021 